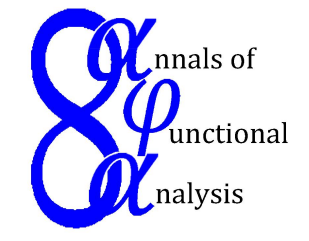

Ann. Funct. Anal. 6 (2015), no. 1, 54-58

http://doi.org/10.15352/afa/06-1-5

ISSN: 2008-8752 (electronic)

http://projecteuclid.org/afa

\title{
ON THE TRANSCENDENTAL RADIUS OF THE VOLTERRA INTEGRATION OPERATOR
}

\author{
LEVON GEVORGYAN
}

Communicated by J. Esterle

\begin{abstract}
The transcendental radius of the Volterra integration operator $V$ acting in the space $L^{2}(0 ; 1)$ is calculated. The latter is compared with the norm of the self-commutator of $V$.
\end{abstract}

\section{INTRODUCTION AND PRELIMINARIES}

Let $A$ be a linear bounded operator, acting in a Hilbert space $(\mathcal{H},\langle\cdot, \cdot\rangle)$. According to [7] there exists a unique complex number $c$ belonging to the closure of the numerical range $W(A)$ such that

$$
m(A)=\inf _{\lambda \in \mathbb{C}}\|A-\lambda I\|=\|A-c I\| .
$$

Fujii and Prasanna [1] called $m(A)$ transcendental radius of $A$. Prasanna proved [6] that

$$
m^{2}(A)=\sup _{\|x\|=1}\left\{\|A x\|^{2}-|\langle A x, x\rangle|^{2}\right\} .
$$

In [5] a more general problem is considered and is proved that

$$
m_{T}^{2}(A)=\left\|T-\lambda_{0} A\right\|^{2}=\inf _{\lambda \in \mathbb{C}}\|T-\lambda A\|^{2}=\sup _{\|x\|=1}\left\{\|T x\|^{2}-\frac{|\langle T x, A x\rangle|^{2}}{\|A x\|^{2}}\right\} .
$$

The number $\lambda_{0}$ is unique if the approximate point spectrum of $A$ does not contain 0 and is characterized by the following conditions. There exists a sequence of unit elements $\left\{x_{n}\right\}$ such that

$$
\left\langle\left(T-\lambda_{0} A\right) x_{n}, A x_{n}\right\rangle \rightarrow 0,\left\|\left(T-\lambda_{0} A\right) x_{n}\right\| \rightarrow\left\|T-\lambda_{0} A\right\| .
$$

Date: Received: May 2, 2013; Accepted: May 22, 2013.

2010 Mathematics Subject Classification. Primary 47A63; Secondary 47A75, 47B47.

Key words and phrases. Minimal norm, transcendental radius, Volterra operator, selfcommutator. 
In [3] is proved that

$$
\lambda_{0}=\lim _{n \rightarrow \infty} \frac{\left\langle T x_{n}, A x_{n}\right\rangle}{\left\|A x_{n}\right\|^{2}},
$$

where $\left\{x_{n}\right\}$ is a sequence of unit vectors, approximating the supremum in (1.2). It is easy to see that (1.4) implies both conditions in (1.3). Indeed, denoting

$$
\lambda_{n}=\frac{\left\langle T x_{n}, A x_{n}\right\rangle}{\left\|A x_{n}\right\|^{2}}
$$

we have

$$
\left\langle\left(T-\lambda_{0} A\right) x_{n}, A x_{n}\right\rangle=\left(\lambda_{n}-\lambda_{0}\right) \cdot\left\|A x_{n}\right\|^{2} \rightarrow 0 .
$$

For the second equality

$$
\left\|T-\lambda_{0} A\right\|^{2}=\lim _{n \rightarrow \infty}\left\{\left\|T x_{n}\right\|^{2}-\left|\lambda_{n}\right|^{2} \cdot\left\|A x_{n}\right\|^{2}\right\} .
$$

On the other hand

$$
\left\|\left(T-\lambda_{0} A\right) x_{n}\right\|^{2}=\left\|T x_{n}\right\|^{2}-2\left\|A x_{n}\right\|^{2} \cdot \operatorname{Re} \lambda_{n} \bar{\lambda}_{0}+\left|\lambda_{0}\right|^{2} \cdot\left\|A x_{n}\right\|^{2} .
$$

\section{MAin RESUlts}

Consider the Volterra integration operator in $L^{2}(0 ; 1)$ defined by the formula

$$
(V f)(x)=\int_{0}^{x} f(t) d t
$$

Easy calculations show that

$$
\begin{aligned}
& \left(V V^{*} f\right)(x)=\int_{0}^{x} t f(t) d t+x \int_{x}^{1} f(t) d t, \\
& \left(V^{*} V f\right)(x)=\int_{0}^{1} f(t) d t-x \int_{0}^{x} f(t) d t-\int_{x}^{1} t f(t) d t .
\end{aligned}
$$

Now we search

$$
\inf _{\lambda \in \mathbb{C}}\|V-\lambda I\|
$$

The equality $V \bar{f}-\bar{c} \bar{f}=\overline{V f-c f}$ implies $\|V-\bar{c} I\|=\|V-c I\|$ and finally, $c \in \mathbb{R}$. Recall ([4], Problem 165) that $W(V)$ is bounded by the curve

$$
t \mapsto \frac{1-\cos t}{t^{2}} \pm i \frac{t-\sin t}{t^{2}}, 0 \leq t \leq 2 \pi
$$

and $\|V\|=\frac{2}{\pi}$, therefore $0 \leqslant c \leqslant \frac{1}{2}, m^{2}(V) \leqslant \frac{4}{\pi^{2}}$.

The operator $S=\left(V^{*}-\lambda I\right)(V-\lambda I)$ is defined by the formula

$$
(S f)(x)=(1-\lambda) \int_{0}^{1} f(t) d t-x \int_{0}^{x} f(t) d t-\int_{x}^{1} t f(t) d t+\lambda^{2} f(x) .
$$


As $V^{*} V-\lambda\left(V^{*}+V\right)$ is a self-adjoint compact operator, its norm coincides with the eigenvalue having the greatest absolute value. For the positive operator $S=$ $V^{*} V-\lambda\left(V^{*}+V\right)+\lambda^{2} I$ one has $\|S\|=\max \{\operatorname{eig}(S)\}$, where $\{\operatorname{eig}(S)\}$ is the set of eigenvalues of $S$.

Calculating the second derivative of (2.1), we get for the eigenfunctions of $S$ the second order differential equation

$$
\left(\mu-\lambda^{2}\right) f^{\prime \prime}(x)+f(x)=0 .
$$

It is easy to see that the eigenfunction satisfies the condition $f^{\prime}(0)=0$, so

$$
f(x)=\cos \frac{x}{\sqrt{\mu-\lambda^{2}}} .
$$

Putting $f(t)=\cos \alpha t$ into (2.1), we get

$$
(S f)(x)=-\frac{\lambda}{\alpha} \sin \alpha-\frac{1}{\alpha^{2}} \cos \alpha+\frac{1}{\alpha^{2}} \cos \alpha x+\lambda^{2} \cos \alpha x,
$$

meaning that $f$ is an eigenfunction corresponding to the eigenvalue

$$
\mu=\frac{1}{\alpha^{2}}+\lambda^{2}
$$

if and only if $\alpha$ satisfies

$$
\cot \alpha+\alpha \lambda=0
$$

For each $\lambda>0$ equation (2.3) has one and only one solution $\alpha$ in each interval $(k \pi+\pi / 2 ;(k+1) \pi), k \in \mathbb{Z}^{+}$, therefore from $(2.2)$ the greatest eigenvalue of $S$ corresponds to the interval $(\pi / 2 ; \pi)$. Then, we have

$$
\mu=\frac{1}{\alpha^{2}}+\frac{\cot ^{2} \alpha}{\alpha^{2}}=\frac{1}{\alpha^{2} \sin ^{2} \alpha} .
$$

The smallest value of $\|S\|$ corresponds to the greatest value of $\alpha \sin \alpha$, which occurs if $\alpha \in(\pi / 2 ; \pi)$ satisfies

$$
\tan \alpha+\alpha=0 \text {. }
$$

From (2.3) and (2.4) we get $\lambda=1 / \alpha^{2}$. So we arrive to the following result.

Proposition 2.1. The transcendental radius of the Volterra integration operator is equal to $\sqrt{1 / \alpha^{2}+1 / \alpha^{4}}$, where $\alpha \in(\pi / 2 ; \pi)$ satisfies (2.4).

The approximate solution of the transcendental equation (2.4), given by MatLab is $\alpha \approx 2.028757838110434 ; \lambda \approx 0.242962685095034$ and

$$
\min _{\lambda}\|V-\lambda I\|^{2} \approx 0.301993551443623 \text {. }
$$

Putting the function $f(x)=2\left(\frac{\alpha}{2 \alpha+\sin 2 \alpha}\right)^{1 / 2} \cos \alpha x$ into (1.4) and (1.1), we get the same values of $\lambda$ and of the minimal norm.

From the general theory of the Sturm-Liouville operator theory the following result may be deduced.

Corollary 2.2. The sequence of function $\left\{\cos \alpha_{k} x\right\}_{k=1}^{\infty}$, where $\left\{\alpha_{k}\right\}$ are the positive roots of the equation $\cot \alpha+\alpha \lambda=0$ form an orthogonal basis of $L^{2}(0 ; 1)$. 
Now we intend to show an application of the result above to a problem in operator theory.

In [7] it is shown that the norm of inner derivation $D_{T}(A)=A T-T A$ is defined by the following formula

$$
\sup _{\|A\|=1}\|A T-T A\|=2 m(T) .
$$

In [2] is proved that for any operator $A$ the self-commutator $C(A)=A A^{*}-A^{*} A$ satisfies the following inequality

$$
\left\|A A^{*}-A^{*} A\right\| \leq\|A\|^{2} .
$$

For some operators the inequality turns to be the equality.

Example 2.3. Let $S$ be the operator of the simple unilateral shift. Then $S^{*} S-$ $S S^{*}$ is the operator of orthogonal projection on the first element of the basis, shifted by $S$, so $\left\|S^{*} S-S S^{*}\right\|=1$ and $\|S\|=1$, hence $\left\|S^{*} S-S S^{*}\right\|=\|S\|^{2}$.

As $C(A-\lambda I)=C(A)$ for any $\lambda \in \mathbb{C}$, inequality (2.5) may be sharpened

$$
\left\|A A^{*}-A^{*} A\right\| \leqslant m^{2}(A) .
$$

For the Volterra operator we have

$$
\left(\left(V^{*} V-V V^{*}\right) f\right)(x)=\int_{0}^{1} f(t) d t-x \int_{0}^{1} f(t) d t-\int_{0}^{1} t f(t) d t .
$$

The self-commutator $C(V)$ is a two dimensional self-adjoint operator with unit eigenfunctions $e_{1}=\sqrt{2+\sqrt{3}}((3-\sqrt{3}) x-1), e_{2}=\frac{1}{\sqrt{2+\sqrt{3}}}((3+\sqrt{3}) x-1)$ corresponding to the eigenvalues $\left\{\frac{\sqrt{3}}{6},-\frac{\sqrt{3}}{6}\right\}$, so

$$
\|C(V)\|=\frac{\sqrt{3}}{6} \approx 0.288675134 \cdots .
$$

Easily can be proved that the operator

$$
(B f)(x)=\frac{1}{\sqrt{2 \sqrt{3}}}((3+\sqrt{3}) x-1) \int_{0}^{1}((3-\sqrt{3}) t-1) f(t) d t
$$

has the same self-commutator $-C(V)=C(B)$.

\section{REFERENCES}

1. M. Fujii and S. Prasanna, Translatable radii for operators, Math. Japon. 26 (1981), 653657.

2. L. Gevorgyan, On operators with large self-commutators, Oper. Matrices 4 (2010), no. 1, 97-104.

3. L. Gevorgyan, On minimal norm of a linear operator pencil, Dokl. Nats. Akad. Nauk Armen. 110 (2010), 653-657.

4. P.R. Halmos, A Hilbert space problem book, D. Van Nostrand Co, Inc, 1967.

5. K. Paul, S.M. Hossein and K.C. Das, Orthogonality on $B(H, H)$ and minimal-norm operator, J. Anal. Appl. 6 (2008), 169-178. 
6. S. Prasanna, The norm of a derivation and the Björk-Thomée-Istratescu theorem, Math. Japon. 26 (1981), 585-588.

7. J. G. Stampfli, The norm of a derivation, Pacific J. Math. 33 (1970), no. 3, 737-747.

Department of Mathematics, State Engineering University of Armenia, YereVAN 0009, ARMENIA.

E-mail address: levgev@hotmail.com 